NBSIR 82-2586

\title{
Development of Power System Measurements -- Quarterly Report April 1, 1982 to June 30, 1982
}

U S. DEPARTMENT OF COMMERCE

Nationai Bureau of Standards

Center for Electronics and Electrical Engineering

Electrosystems Division

Vashington, DC 20234

October 1982

Prepared ior:

Department of Energy

Division of Electric Energy Systems

- $Q \mathrm{C}$ Independence Avenlie, S.W.

100 ington, DC 20585 

DEVELOPMENT OF POWER SYSTEM

MEASUREMENTS -- QUARTERLY REPORT

APRIL 1, 1982 TO JUNE 30, 1982

R. E. Hebner, Editor

U.S. DEPARTMENT OF COMMERCE

National Bureau of Standards

Center for Electronics and Electrical Engineering

Electrosystems Division

Washington, DC 20234

October 1982

Prepared for:

Department of Energy

Division of Electric Energy Systems

1000 Independence Avenue, S.W.

Washington, DC 20585

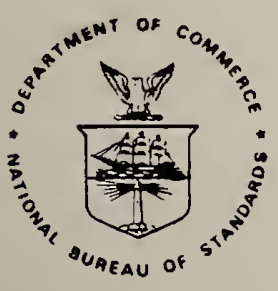

U.S. DEPARTMENT OF COMMERCE, Malcoim Baidrige, Secretary NATIONAL BUREAU OF STANDARDS, Ernest Ambler, Director 



\section{Foreword}

This report is intended to summarize the progress on four technical investigations during the third quarter of FY 1982. Although reasonable efforts have been made to ensure the reliability of the data presented, it must be emphasized that this is an interim report so that further experimentation and analysis may be performed before the conclusions from any of these investigations are formally published. It is, therefore, possible that some of the observations presented in this report will be modified, expanded or clarified by our subsequent research. 
Foreword .......................... i . . . . . . LIST OF FIGURES .....................

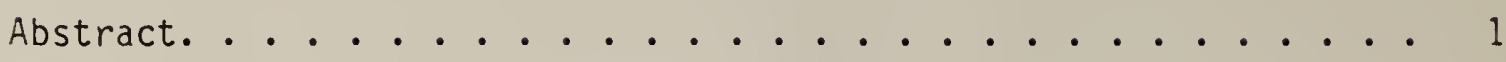

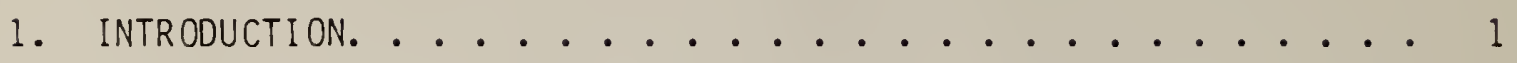

2. DC FIELDS AND ION MEASUREMENTS

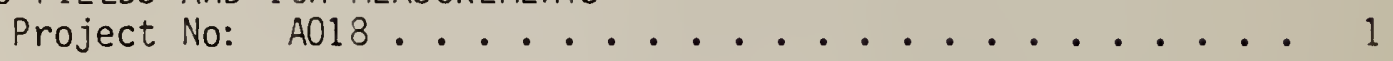

3. INCIPIENT FAULT DETECTION/LOCATION

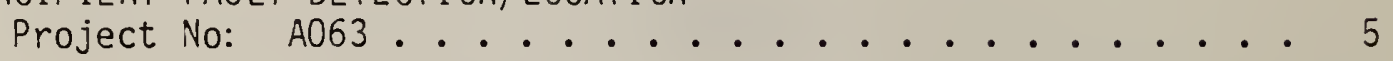

4. TECHNICAL ASSISTANCE FOR FUTURE INSULATION SYSTEMS RESEARCH Project No: A053 ................. 7

5. OPTICAL MEASUREMENTS FOR INTERFACIAL CONDUCTION AND BREAKDOWN IN INSULATING SYSTEMS

Project No: A057 . . . . . . . . . . . 15

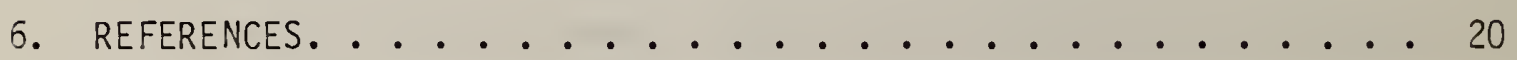


Figure 1. Measured space charge density for three different flow rates as a function of ion counter voltage (relative to ground). Data are for flow rates of $16.5 \times 10^{-3} \mathrm{~m}^{3} / \mathrm{s}-\square$; $4.4 \times 10^{-3} \mathrm{~m}^{3} / \mathrm{s}-0 ; 2.5 \times 10^{-3} \mathrm{~m}^{3} / \mathrm{s} \ldots \ldots 3$

Figure 2. Comparison of space charge density measurements for front and top inlets to the ion counter. The flow rate was $16.5 \times 10^{-3} \mathrm{~m}^{3} / \mathrm{s}$. Top opening - $\square$;

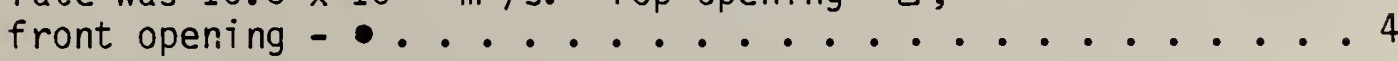

Figure 3. Experimental arrangement for investigating the effect of radiation on discharge initiation in $\mathrm{SF}_{6} \ldots \ldots . . . .9$

Figure 4. Voltage dependence of fractional differences between electron avalanche count rates recorded by the in-phase and out-of-phase counters for a chopped uv beam irradiating the tip of the point electrode and for no irradiation. The wavelength band of the radiation was from 295 to $575 \mathrm{~nm}$; the gas pressure was $200 \mathrm{kPa}$; and the point radius of curvature and gap spacing were $0.71 \mathrm{~mm}$ and

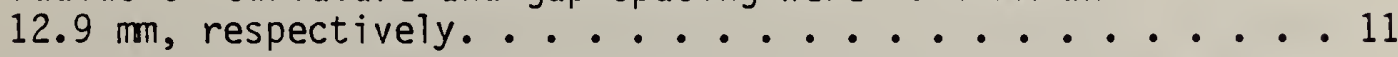

Figure 5. Voltage dependence of count rates recorded by the in-phase and out-of-phase counters for filtered and unfiltered, chopped uv beams irradiating

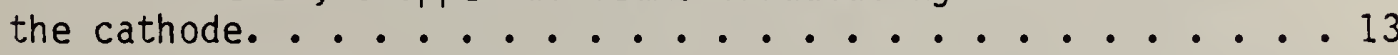

Figure 6. Electrode geometry with pressboard interface as viewed from the detector. This is an end view of the $\sim 30 \mathrm{~cm}$ plates with a pressboard interface extending $28 \mathrm{~cm}$ of the length. The notch in the electrode serves to produce almost zero field region. The light intensity as a function of position along different tracks $A, B$, and $C$ can be measured by the detector and compared with one another to obtain relative electro-optical field measurements. . . . . . 16

Figure 7. Computer calculation of the equipotentials for a notch in a parallel-plate electrode system assuming no space charge. The field is seen to be unaffected along the plate opposite the notch and essentially zero at the interior of the notch. The inset shows higher resolution of equipotentials where the dotted equipotentials are the ones shown in the main drawing. . . . 17 


\section{LIST OF FIGURES (cont)}

Page

Figure 8. Comparison of electric field next to the interface and away from the interface in nitrobenzene dopped-transformer oil. Although there is an obvious, negative-spacecharge-induced field distortion which is spread somewhat uniformly throughout the gap, the field distortion is not different near the interface from what it is away from the interface (within 10\%) .......... 19 


\section{DEVELOPMENT OF POWER SYSTEM MEASUREMENTS -- QUARTERLY REPORT April 1, 1982 TO June 30, 1982 \\ R. E. Hebner, Editor}

This report documents the progress on four technical investigations sponsored by the Department of Energy and performed by the Electrosystems Division, the National Bureau of Standards. The work described covers the period April 1, 1982 to June 30, 1982. This report emphasizes the errors associated with measurements of space charge near dc transmission lines, the measurement of rf attenuation in distribution cables, the characteristics of positive and negative corona in compressed $\mathrm{SF}_{6}$ gas, and the measurement of the space charge density in transformer oil subjected to 60-Hz excitation.

Key words: cables; dc fields; high voltage; incipient fault; insulation; $\mathrm{SF}_{6}$; space charge; transformer oil.

\section{INTRODUCTION}

Under an interagency agreement between the U. S. Department of Energy and the National Bureau of Standards, the Electrosystems Division, NBS, has been providing technical support for DOE's research on electric energy systems. This support has been concentrated in four areas -- the measurement of electric fields, the measurement of the electromagnetic properties of solid insulating materials and cables, the measurement of interfacial electrostatic field distributions, and of space charge density. The technical progress made during the quarter April 1 to June 30, 1982 is summarized in this report.

\section{DC FIELDS AND ION MEASUREMENTS Project No: A018}

The objectives of this effort are to investigate devices and measurement techniques which may be used to characterize the electrical environment near high-voltage dc (HVDC) transmission lines, to evaluate methods being used for calibrating such devices, and to develop and establish calibration facilities at NBS which will permit independent verification of the accuracy of user calibrations.

By developing appropriate methods of instrument calibration, it will be possible to compare measurements taken in the vicinity of a high-voltage transmission line with measurements taken in biological exposure facilities. such a comparison will be necessary in any formal or informal risk assessment.

During this quarter, primary effort has been directed at the problem of measuring space charge densities near a HVDC transmission line by using an ion counter. These studies are a continuation of those done previously [1]. 
The purpose of these studies is to determine the effect of the large electric fields existing near a HVDC transmission line on measurements of ion density. Ion density is measured by using an ion counter, which is an aspiration device. Air containing ions is drawn into the ion counter and ions are precipitated onto collector plates by an internal electric field. Near the inlet of the ion counter, ions are subjected to competing forces: the hydrodynamic forces of the air flowing into the counter and the electric forces due to the external electric field. If the speed of the ions due to the electric field is much larger than that due to the air flow, then it can be expected that ions are driven to the counter walls, rather than entering the air stream. In these studies, the effects of varying the air speed at the inlet and the potential of the ion counter assembly were investigated. (The potential referred to here is that of the overall counter system, not the voltage that exists inside the counter to remove the ions from the air stream).

A monopolar line $2 \mathrm{~m} \mathrm{high}$ and $6 \mathrm{~m}$ long was erected in an open space in a high bay area to minimize the effect of surrounding objects. An ion counter and associated electronics were arranged so that the potential of the counter could be varied from $+25 \mathrm{kV}$ to $-25 \mathrm{kV}$. In addition, an extension was placed on the counter with both front-and top-facing openings. For the tests reported here, the ion counter inlet was located under the center of the line and the top of the counter was approximately $0.43 \mathrm{~m}$ above ground. The flow rate through the ion counter could be varied by approximately a factor of 10. By using this system, it is possible to investigate the effects of ion counter voltage, geometry, and flow rate on indicated ion density. Here geometry refers both to the ion counter position and the location of the inlet.

Electric field and ion current density were measured under the monopolar line. It was found that both electric field $E$ and current density $J$ were uniform to within $\sim \pm 10$ percent as the measurement position was varied in a direction parallel to the line. In the transverse direction, measurements showed that both $E$ and $J$ peaked under the line as expected. For a $l$ ine voltage of $-60 \mathrm{kV}$, the electric field under the 1 ine was approximately $-30 \mathrm{kV} / \mathrm{m}$. For a fixed line voltage, ion counter flow rate, and geometry, the spacecharge density was determined as a function of the ion counter voltage. These measurements were repeated for different flow rates, geometries, and line voltages.

An example of the results obtained, figure 1 , shows the measured space-charge density $\rho$ as a function of the voltage of the ion counter assembly for three different flow rates. Clearly, the space-charge density depends on the flow rate. Also, the indicated ion density drops significantly as the voltage approaches $-20 \mathrm{kV}$. These data were obtained with an inlet on the top of the ion counter housing and $-60 \mathrm{kV}$ on the line.

The effects of inlet location are shown in figure 2. Plotted here are the measured ion densities as a function of ion counter voltage for a top opening and a front opening. The air flow was the same for the two measurements and line voltage was $-60 \mathrm{kV}$. 


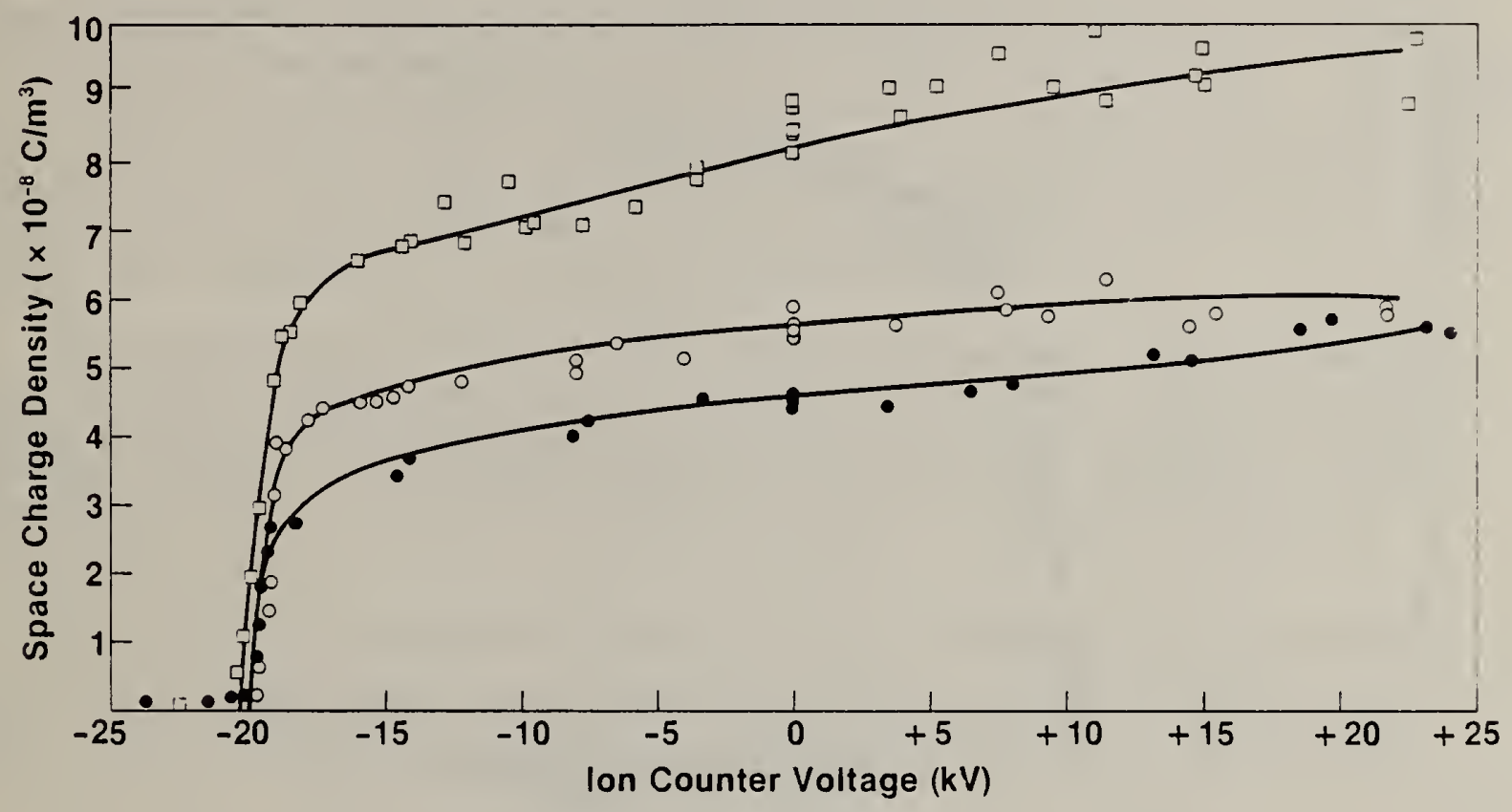

Figure 1. Measured space charge density for three different flow rates as a function of ion counter voltage (relative to ground). Data are for flow rates of $16.5 \times 10^{-3} \mathrm{~m}^{3} / \mathrm{s}-0$; $4.4 \times 10^{-3} \mathrm{~m}^{3} / \mathrm{s}-0 ; 2.5 \times 10^{-3} \mathrm{~m}^{3} / \mathrm{s}-\bullet$. 


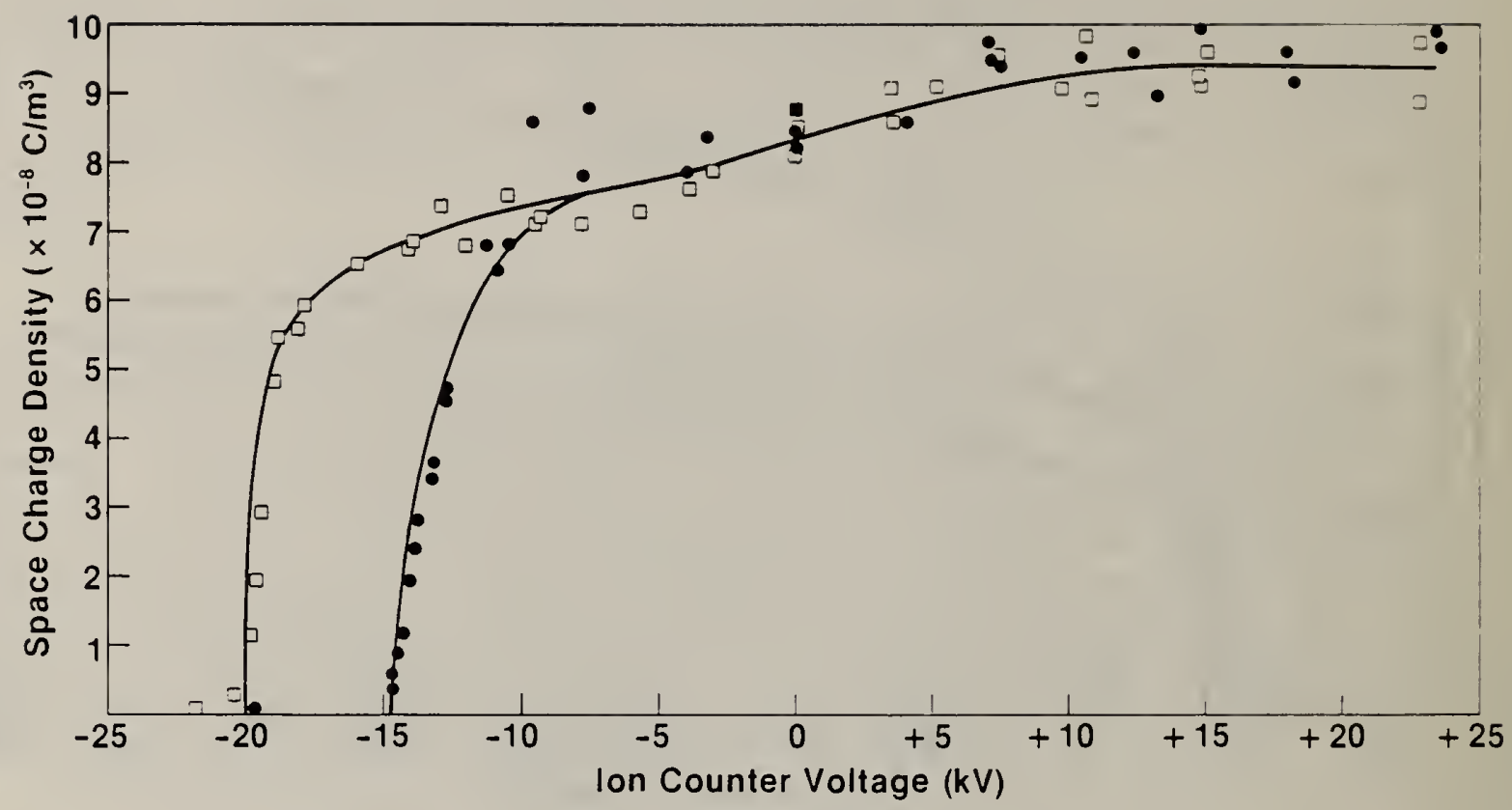

Figure 2. Comparison of space charge density measurements for front and top inlets to the ion counter. The flow rate was $16.5 \times 10^{-3} \mathrm{~m}^{3} / \mathrm{s}$. Top opening - $\square$; front opening - $\bullet$. 
Space potential near the counter inlet was measured using an electrostatic voltmeter. It varied from 10.2-13.5 kV over the vertical extent of the front opening. By contrast, the ion counter assembly assumed a potential of $-20 \mathrm{kV}$ when allowed to float above ground under the same conditions. These observations are consistent with the theory of charging of a particle in a uniform field with a unipolar ion flux which predicts an isolated object will equilibrate at a potential greater than the unperturbed midplane potential [2].

Two questions arise from this (and previous) work. The first has to do with attempting to bias an ion counter to space potential to minimize measurement errors. The second, and more important one for present day interest, concerns the flow dependence of the measured space charge density. Estimates of losses in the ion counter assembly due to space charge effects give results which are too low to explain the observations. It appears more likely that the strong electric fields near the ion counter cause a loss of ions to the counter inlet. This effect becomes less important as the hydrodynamic forces become more important, i.e., the flow rate increases.

Many questions have been raised concerning ion density measurements under a HVDC transmission line as a result of this work. Future work will concentrate on resolving the cause of the flow rate dependence and the geometry effect. Some computer modeling will be done, although it will be necessary to use a greatly simplified model of the actual physical situation.

For further information, contact Dr. R. H. McKnight, (301) 921-3121.

\section{INCIPIENT FAULT DETECTION/LOCATION Project No: A063}

The objective of this program is to identify and, insofar as practical, remove technical barriers to the development of a successful incipient fault detector/locator for underground power transmission use. NBS responsibility includes conducting an experimental program which will aid the development of an incipient fault detection/location system by measuring the rf characteristics of power cables and evaluating the frequency content of partial discharge pulses emanating from incipient fault sites in a cable dielectric. The measurement program at NBS is now completed. The limitations of time-domain reflectometry (TDR) techniques for detecting incipient fault sites in transmission cables have been determined. The radio frequency ( $r f$ ) responses of these cables have also been measured. This quarter the studies of the effect of sampling rate drift on transfer function measurements have been completed.

In order to measure the transfer function of a power cable using TDR techniques, two waveforms are required (e.g., the input and output waveforms or the output waveforms from two different lengths of cables). In our measurements, the two time-domain waveforms (shaped like step functions) are Fourier transformed to the frequency domain and their ratio yields the transfer function. In our early efforts, we noted that when making very precise measurements ( $i . e ., \pm 0.1 \mathrm{~dB}$ ), the measured transfer function often showed a steady gain or roll-off with frequency. The fact that a gain implied more energy was leaving the cable than entering it suggested 
that something was wrong with the measurement approach. The problem was traced to sampling rate drift in the digitizing oscilloscope. If the sweep speed changes between the measurement of the two waveforms, then the time interval between successive samples will change. Such a drift in sampling rate can be shown to yield a similar drift in the transformed frequency domain transfer function.

One approach to solving this problem is to apply a correction in the frequency domain. That is, if the measured transfer function shows a rolloff or gain with frequency, multiply it by a suitable frequency-dependent correction-factor to remove the effect. In order to do this, one must be certain that the transfer function is supposed to be flat. One must, therefore, have prior knowledge of the transfer function before the measurement. For that reason, this approach seems less than satisfactory.

As described last quarter, our approach has been to correct the data in the time domain. In our measurements, the first 100 or so points of the sampled time-domain waveforms should be the same for both measured waveforms since these data points represent the measured response of the cables and connectors between the TDR unit and the cable being measured. If no sampling rate drift has occurred, these points will be identical. By contracting or expanding the sampling interval of the second waveform with the aid of Tinear or cubic interpolation, the first 100 points of the second waveform should align with the first 100 points of the first waveform. There is one major complication, however. The two waveforms are often horizontally displaced with respect to one another (i.e., the "step" does not occur at the same horizontal position on the oscilloscope screen). Our approach was to do a least squares fit of the two waveforms by first sliding the second waveform left or right until the correct horizontal displacement was determined, and then doing a least squares fit by expanding or contracting the sampling interval of the second waveform to agree with the first. Unfortunately, we found that these two operations are not independent (i.e., the best fit sampling drift correction is dependent on the horizontal displacement correction and vice versa).

In order to do a least squares fit with two dependent variables, we implemented a version of the Marquart algorithm available on the main NBS computer. This software worked very well in quickly finding correction parameters yielding a better fit than our previous approach. The resulting transfer functions from these calculated correction parameters appeared to be free of the effect of sampling rate drift. Unfortunately, the signal-to-noise ratio was decreased for the transfer functions derived from the corrected waveforms. This may be a result of the interpolation scheme used to create the corrected waveforms, although we attempted to make the interpolation as mathematically smooth as possible.

We have shown that sampling rate drift can affect the results of precision transfer function measurements in a predictable manner. This by itself is an important contribution. We have, also, demonstrated that this drift may be corrected for in the time domain without the a priori knowledge which is required for a freqnency-domain correction. Cur results indicate, however, that a perfect correction for sampling rate drift is difficult if not impossible (i.e., the corrected waveform has a significantly lower signal-to-noise ratio). 
This suggests that the experimenter should attempt to avoid the problem if at all possible as opposed to attempting to correct for it in software. This might be accomplished by using an oscilloscope with a more stable sweep speed or using a stable time source to calibrate the oscilloscope sweep speed for each acquired waveform.

For further information, contact Dr. W. E. Anderson, (301) 921-3121.

\section{TECHNICAL ASSISTANCE FOR FUTURE INSULATION SYSTEMS RESEARCH Project No: A053}

The objective of this project is to develop diagnostic techniques to monitor, identify, and predict degradation in future compressed gas electrical insulating systems under normal operating conditions. The focus is on the fundamental information and data needed to improve test, design, and performance evaluation criteria. The investigation of partial discharges (corona) in gaseous dielectrics is emphasized. This phenomenon gives rise to degradation of the gas under high electrical stress and may lead to breakdown. Measurement of partial discharge inception in highly nonuniform fields may prove to be a preferred method to determine dielectric strength of electronegative gases.

Planned activities for FY 82 include: 1) completion of an archival paper on basic mechanism of corona inception in SF 6 under ac and dc conditions; 2) investigation of the wavelength dependence of photon-induced positive corona inception in $\mathrm{SF}_{6} ; 3$ ) continuation of measurement of power dependence of the rates of oxyfluoride and water vapor production from corona discharges in $\mathrm{SF}_{6}$ and report the results in a conference and an archival paper; 4) exploration of the feasibility of new methods to measure field-enhanced collisional detachment of negative ions in $\mathrm{SF}_{6}$ and other electronegative gases; 5) improvement of the accuracy of quantitative analysis of trace gases such as $\mathrm{H}_{2} \mathrm{O}$ in $\mathrm{SF}_{6}$ and other gaseous dielectrics using a gas chromatograph/mass spectrometer (GC/MS), and to extend the measurements of the effects of trace $\mathrm{H}_{2} \mathrm{O}$ on corona characteristics and relative dielectric strength of the gas; and 6) completion of an archival paper on compilation and evaluation of electron swarm data in molecular electronegative gases.

Activities 1) and 6) were completed in previous quarters. During the past reporting period, measurements were performed which essentially brought activity 2) to completion. The results obtained from these measurements will be highlighted in this report.

Progress was also made on activities 3 ) and 5 ). A paper entitled "Effects of $\mathrm{H}_{2} \mathrm{O}$ on the Behavior of $\mathrm{SF}_{6}$ Corona," by R. J. Van Brunt was accepted for inclusion in the Proceedings of the Seventh International Conference on Gas Discharges and Their Applications, which was held in London, August 31 - September 3, 1982. Information was acquired on various standard and common methods used to quantitatively measure moisture content in static SF 6 gas samples. It was decided that a calibrated, thin film, aluminum oxide hygrometer probe should be purchased which can be used simultaneously with the present gas chromatograph/mass spectrometer and 
serve as a secondary standard for calibration of the GC/MS for cases where $\mathrm{H}_{2} \mathrm{O}$ is the only contaminant present in $\mathrm{SF}_{6}$. The GC/MS could then be used to evaluate the performance of the hygrometer probe when other gaseous polar contaminants are present, such as generated during an arc or corona discharge.

A means of interfacing the NBS GC/MS data processor system to external computers was established during the past reporting period. This will allow more efficient and rapid handling of GC/MS data acquired from quantitative measurements of corona-induced end decomposition products in gaseous dielectrics. It also gives more flexibility to the data analysis procedure. Development of new software for GC/MS data analysis is currently in progress.

Major emphasis during this quarter was given to performing measurements relevant to activity 2) on investigation of the wavelength dependence of photon-enhanced electron avalanche initiation around a positive point electrode in pressurized SF6. Previous measurements performed in our laboratory have shown $[3,4]$ that initiation of electron avalanches near inception of positive point-plane corona in SF 6 can be dramatically enhanced by exposing the region near the tip of the point electrode to radiation from an $\mathrm{Hg}$-discharge lamp in the range of 295 to $750 \mathrm{~nm}$. It was speculated that this enhancement was due to an increase in the rate of electron release near the point by the mechanism of photodetachment from negative ions, e.g.,

$$
h v+S F_{6}^{-} \rightarrow S F_{6}+e .
$$

An increase in the probability of discharge initiation for positive dc and impulse breakdown in $\mathrm{SF}_{6}$ when uv-radiation is directed at the more highly stressed electrode has also been observed by others [4].

The experiment described here was designed to determine the relative importance of photodetachment as an electron release mechanism near a positive point electrode in $\mathrm{SF}_{6}$ and other electronegative gases. Up to the present time results have been obtained in our laboratory only for $S_{6}$.

A diagram of the experimental setup is shown in figure 3. The plane electrode (cathode) could be uniformly irradiated with uv-radiation from an $\mathrm{Hg}$-discharge lamp, the output of which was predominantly at a wavelength of $253.7 \mathrm{~nm}$. This radiation was used to control the negative ion density in the interelectrode gap through release of low-energy photoelectrons that immediately attach to SF6 molecules in the gas.

The region near, and including, the tip of the point electrode could be irradiated with a chopped and collimated beam from either a tunable dye laser with a range from 570 to $630 \mathrm{~nm}$ or a high-power, broadband Hg-discharge lamp with output in the range of 750 to $295 \mathrm{~nm}$. The latter source was used to produce the effect previously reported $[3,4]$, although in those experiments, no attempt had been made to insure that the beam was sufficiently collimated to avoid hitting surfaces other than the point electrode, e.g., the cathode surface. The purpose of the beam directed at the point was to induce photodetachment. With the use of the chopper and detection scheme shown in figure 3 , it was possible to measure the difference between the 


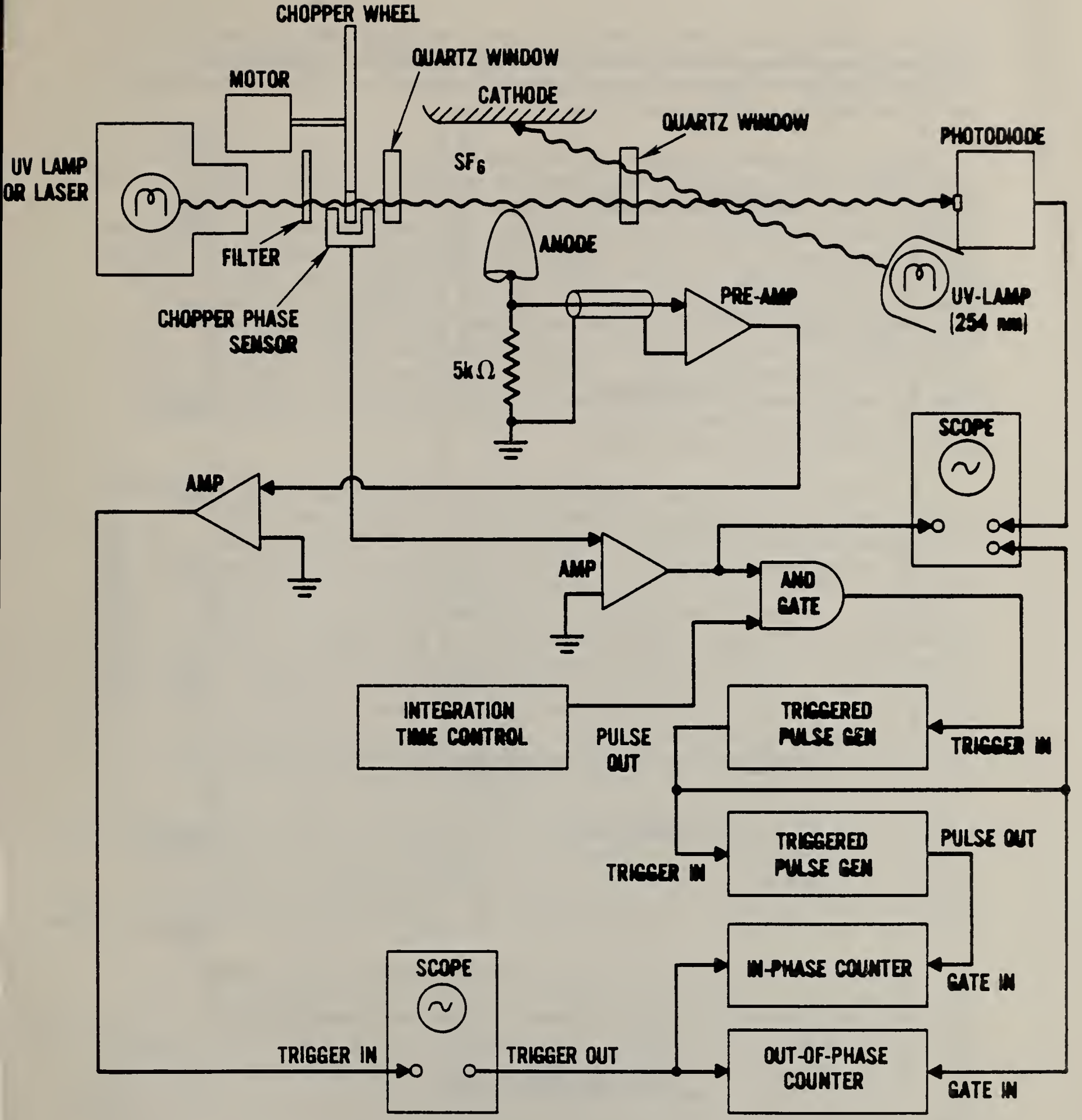

Figure 3. Experimental arrangement for investigating the effect of radiation on discharge initiation in $\mathrm{SF}_{6}$. 
rates of electron avalanche formation for irradiated and unirradiated conditions near the point. The count rates for the irradiated and unirradiated point electrode were recorded respectively by the in-phase and out-of-phase counters indicated in figure 3.

When the $\mathrm{Hg}$-lamp was used, it was possible to restrict the band of wavelength of the incident radiation with optical filters. Otherwise, the radiation was simply transmitted through a quartz window. Both light sources could be blocked in order to check the background avalanche rates. It was also possible to direct the collimated beam to locations in the gap other than the tip of the point electrode. All avalanches with a charge level in excess of $0.02 \mathrm{pC}$ were detected and recorded by the counters. The trigger level of the oscilloscope served as a common discriminator for both counters. Care was taken to eliminate phase sensitive noise, e.g., pickup from the chopper motor. Polished stainless steel electrodes were used and measurements were performed for gap spacings of 12.9, 12.2, and $13.5 \mathrm{~mm}$, with corresponding point electrode radi $i$ of curvature of 0.720 , 0.475 , and $0.043 \mathrm{~mm}$, respectively.

The results for $\mathrm{SF}_{6}$ at pressures in the range between 100 and $300 \mathrm{kPa}$ indicate that photodetachment of negative ions plays a very minor role in the initiation of electron avalanches near a positive electrode. This process, therefore, cannot be invoked to explain the photo-enhancement of electron avalanche rates previously reported $[3,4]$. In all cases studied, less than 5 percent of the observed avalanches could be attributed to an interaction of the light beam with the gas in the immediate vicinity of the positive electrode. In most cases, no statistically significant signals were observed. Weak evidence of photodetachment was sometimes seen, but only at voltages close to the threshold for avalanche formation and only for wavelengths shorter than $350 \mathrm{~nm}$. An example of a typical result is shown in figure 4. Plotted in this figure are the fractional differences in the count rates recorded by the in-phase and out-of-phase counters as a function of applied voltage for unfiltered uv-radiation, i.e., $\left(N_{1}-N_{2}\right) /\left(N_{1}+N_{2}\right)$, where $N_{1}$ and $N_{2}$ are the accumulated counts from the in-phase and out-of-phase counters, respectively. The error bars represent the calculated uncertainties, namely, $\pm 2 \sigma$, where $\sigma=\left(N_{1}+N_{2}\right)^{-1 / 2}$. Points above the axis that lie outside the error bars are considered to be statistically significant. Also shown by the open symbols are data obtained when the incident beam was blocked.

Because the cross sections for photodetachment of negative ions are known to be relatively small (< $\left.10^{-16} \mathrm{~cm}^{2}\right)[5]$, it is perhaps not too surprising that the contributions from this process were found to be negligible. Although the cross sections for photodetachment of $\mathrm{SF}_{6}{ }^{-}$and its clusters with other molecules, in particular $S_{6}$, are not known at the present time, there is no a priori reason to expect the cross sections for these molecular ions to differ appreciably from those of other species for which data exist. Moreover, recent calculations [7] of the relative energies of $\mathrm{SF}_{5}{ }^{-}$and $\mathrm{SF}_{5}$ electronic states have yielded a photodetachment threshold of $3.0 \mathrm{eV}$ for $\mathrm{SF}_{6}{ }^{-}$: If this is true, then one would not expect to observe photodetachment for incident radiation with wavelengths shorter than $400 \mathrm{~nm}$. For the unfiltered radiation source used, about 47 percent of the radiative intensity is due to wavelengths shorter than this value, and most of this is concentrated in the range 355-365 $\mathrm{nm}$. For wavelengths shorter than $350 \mathrm{~nm}$ the maximum 


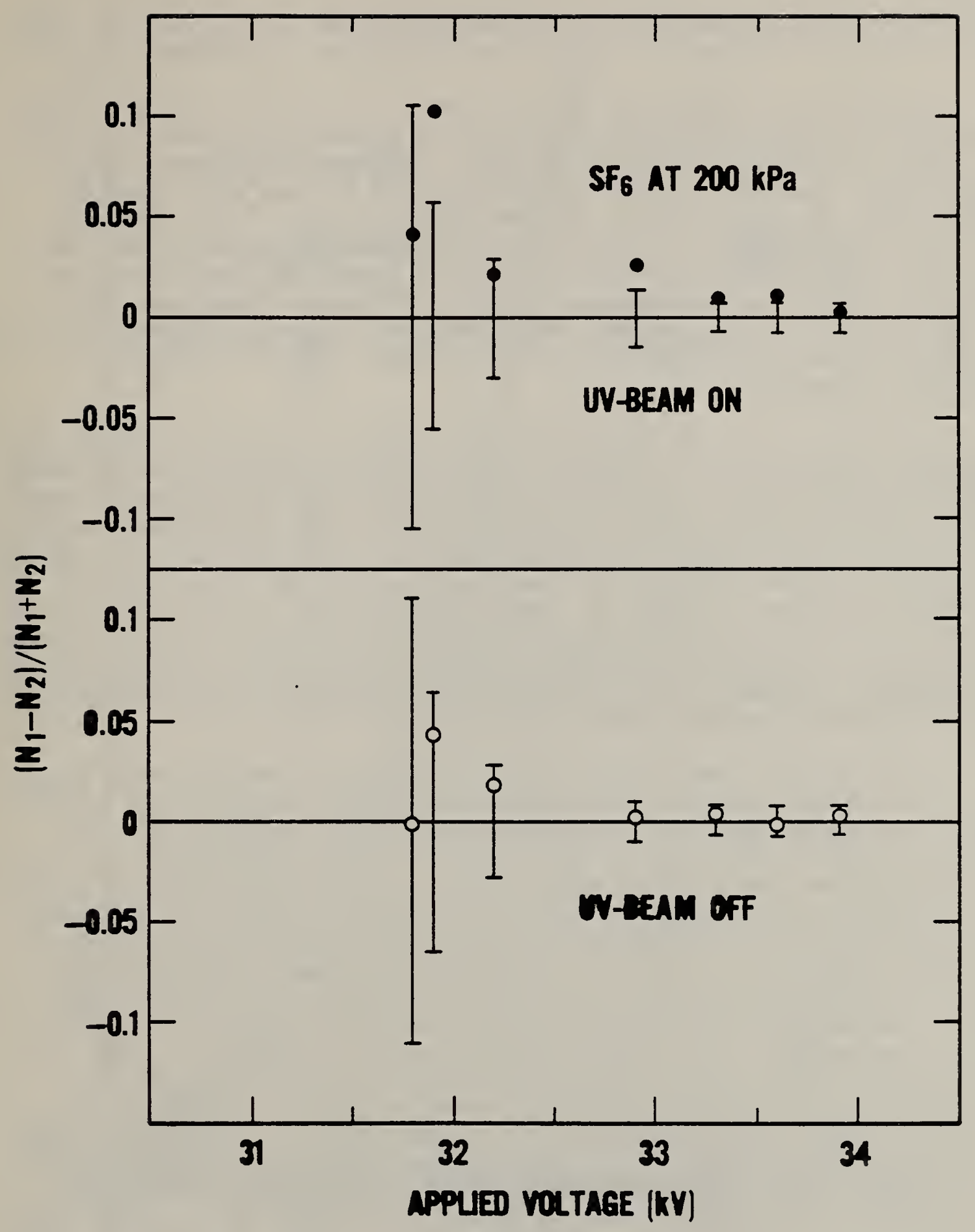

Figure 4. Voltage dependence of fractional differences between electron avalanche count rates recorded by the in-phase and out-of-phase counters for a chopped uv beam irradiating the tip of the point electrode and for no irradiation. The wavelength band of the radiation was from 295 to $575 \mathrm{~nm}$; the gas pressure was $200 \mathrm{kPa}$; and the point radius of curvature and gap spacing were $0.71 \mathrm{~mm}$ and $1.29 \mathrm{~mm}$, respectively. 
obtainable intensity is less than $140 \mathrm{~mW} / \mathrm{steradian.} \mathrm{It} \mathrm{thus} \mathrm{appears} \mathrm{that}$ there is insufficient photon flux in the range below $400 \mathrm{~nm}$ from the light source used here to observe a significant effect due to photodetachment.

The previously observed effect of uv-radiation on electron avalanche enhancement in SF6 was probably the result of photoelectron release from the cathode surface by scattered radiation. As previously noted, these electrons are expected to be of low energy $(<0.5 \mathrm{eV})$ and will therefore readily attach to gas molecules to form negative ions. These ions will drift into the high field region near the point electrode where they may then acquire sufficient translational kinetic energy to allow detachment to occur via collisions with gas molecules. It therefore appears that when radiation is present at the intensities used in these experiments, the dominant electron release mechanism for avalanche initiation is still collisional detachment.

If, as seems to be the case here, the effect of the radiation is merely to increase the negative ion density in the gap, the optimum enhancement in the avalanche rate should be achieved by directing the chopped uv-beam at the cathode surface rather than at the point. Some measurements were performed for a configuration where the gap was irradiated only by a collimated beam directed at the cathode. An example of results obtained from such an experiment are shown in figure 5. These indicate that if an optical filter is used which eliminates wavelengths shorter than $350 \mathrm{~nm}$, no avalanching is observed to take place over the range of voltages shown. Thus, photons with energies in excess of $3.5 \mathrm{eV}$ are apparently needed for photoelectron ejection from the stainless steel electrode.

It is also seen from figure 5 that when photons with an energy between 3.5 and $4.2 \mathrm{eV}$ are allowed to impinge on the cathode, electron avalanches appear at voltages below $30 \mathrm{kV}$ only when the uv-light beam is on, i.e., only during the in-phase condition. Above $30 \mathrm{kV}$ avalanches also begin to appear during the out-of-phase condition, i.e., when the uv-beam is cut off. For this particular measurement there was a delay of about 1.0 ms between the time that the uv-beam was cut off and the time that the out-of-phase counter was gated on. It appears that when the number of negative ions created in avalanches during the uv exposure becomes sufficiently high, there is reasonable probability that some ions will remain in the gap to initiate avalanches 1 ms or more after the beam is turned off. This is not unexpected since our previous calculations [4] based on known ion mobilities have shown that ion transit times can be up to $\sim 1$ ms for gap field conditions such as existed for this measurement. Furthermore, some reduction in the electric field in the gap which will cause an increase in ion residence times can be expected if the ion space charge is allowed to build up during avalanching. On the other hand, the effect observed here may be at least in part due to interactions of positive ions or metastables with the cathode which cause release of secondary electrons even if the radiation is turned off. Further studies of these "delayed" avalanches might be of interest because of the information that can possibly be extracted about ion transit times as well as times required for ion space charge to clear the gap.

The results reported here indicate that in the measurement of pointplane breakdown in SF6 or other highly electronegative gases, a greater 


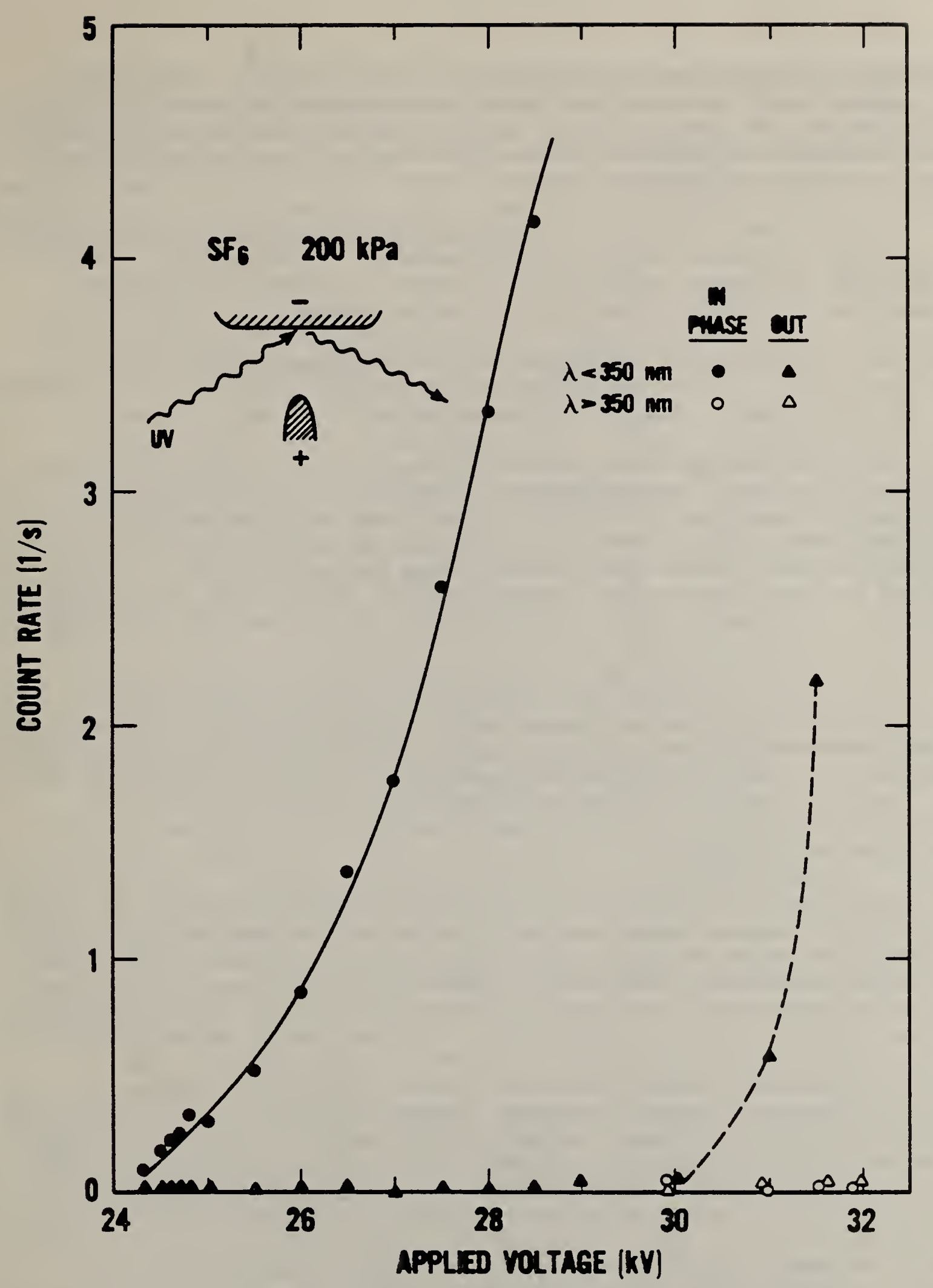

Figure 5. Voltage dependence of count rates recorded by the in-phase and outof-phase counters for filtered and unfiltered, chopped uv beams irradiating the cathode. 
enhancement in the breakdown probability can be achieved by irradiating the cathode rather than the point electrode as is now the common practice. This suggestion of course only applies to situations such as existed for the measurements discussed here where the radiation is not of sufficient energy to cause ionization of the gas.

During the next quarter, the measurements of the effect of uv-radiation on discharge initiation in $\mathrm{SF}_{6}$ will be completed and extended to include $\mathrm{O}_{2}$ and $\mathrm{O}_{2} / \mathrm{N}_{2}$ mixtures. Again, for these gases an attempt will be made to determine the possible importance of photodetachment in initiation of avalanches in an irradiated gap. The photodetachment cross sections and thresholds are well known for negative ions from oxygen. Thus, there is some hope in this case of predicting the contribution to avalanche formation from photodetachment. Some of the measurements on SF 6 may be repeated with a more intense uv source. More measurements will be performed to determine the discharge power dependence of gaseous by-product production rates in SF6 corona. The development of software for analyzing GC/MS data should be completed. This will allow a more rapid and efficient handling of data. Improvements in the methods of calibrating the GC/MS will be explored. A thin film aluminum oxide hygrometer probe will be acquired for use in performing in-situ absolute calibrations of the GC/MS response to trace water vapor content in $\mathrm{SF}_{6}$. By this method, it should be possible to calibrate the GC/MS for $\mathrm{H}_{2} \mathrm{O}$ concentrations down to $10 \mathrm{ppm}$.

It is expected that the results of measurements performed here to investigate effects of irradiation on discharge initiation will prove useful in designing tests to measure the dielectric strengths of electronegative gases used for insulating high-voltage systems. To achieve conditions of gap irradiation which optimize the probability for breakdown requires a better understanding of the mechanisms responsible for discharge initiation when radiation is present.

The effects of contaminants, such as water vapor and air, on the electrical properties of gaseous dielectrics are also not well understood, particularly for conditions where these are present only at trace levels. These effects need to be better understood from a fundamental point-of-view before one can decide on meaningful standards or requirements for gas purity under various practical operating conditions. This question is quite important in the case of $\mathrm{H}_{2} \mathrm{O}$ contamination of $\mathrm{SF}_{6}$ which is now commonly used in insulating systems. Water vapor which can be introduced during gas handling even under the most careful procedures is very difficult to avoid. Although $\mathrm{H}_{2} \mathrm{O}$ vapor is known to affect the static, uniform field dielectric strength of $\mathrm{SF}_{6}$ only slightly, there is evidence that it affects impulse breakdown, the intensity of corona, and the rate of gaseous decomposition during a discharge. The influence of water on the statistics of discharge initiation in gaseous dielectrics needs to be further investigated as a function of controlled water concentration in order to isolate and understand its effects on time-to-breakdown under impulse conditions and on the growth of electron avalanches and streamers in nonuniform fields. Modeling of avalanche growth in water-contaminated $\mathrm{SF}_{6}$ appears feasible and should be considered for the future. Methods for measuring trace levels of water in $\mathrm{SF}_{6}$ and other gaseous dielectrics need to be evaluated, particularly under conditions where other polar contaminant species may be present. 
Improvements need to be made in techniques for analyzing quantitatively the presence of gaseous contaminants in gas insulation at trace levels. This is important for the experiments designed to measure the rates of production of decomposition products under a wide range of discharge conditions. More reliable quantitative data are needed on these rates before tests can be designed which use gas analysis as a diagnostic technique for fault detection in practical gas insulated power systems.

For further information, contact Dr. R. J. Van Brunt, (301) 921-3121.

5. OPTICAL MEASUREMENTS FOR INTERFACIAL CONDUCTION AND BREAKDOWN IN INSULATING SYSTEMS

Project No: A057

The objectives of this investigation are to develop apparatus and appropriate procedures for the optical measurements of interfacial electric field and space-charge density in materials for electric power equipment and systems, to understand the interfacial prebreakdown and breakdown processes in specific insulating systems, and to demonstrate the applicablity of the instrumentation developed and the procedures in the development and design of future systems.

Previous electro-optical measurements of the electric field in transformer 0 il were confined to uniform fields without the presence of an interface. Such data provided an understanding of space charge in transformer oil. It was found that space charge was present in quantities which would change the uniform field by more than 10 percent only when the temperature of the $0 i 1$ exceeded $100^{\circ} \mathrm{C}$.

This quarter's activities centered on examining the electric field in the presence of an interface. Figure 6 shows the electrode geometry and a view of the detector. The scanning characteristics of the two-dimensional detector permit the isolated viewing of different portions of the interelectrode region. In this way, electro-optical electric field measurements as a function of position may be made for different locations in the gap. One electrode is notched along its length parallel to the light beam in order to provide a nearly zero-field region. This notch provides a means to check the accuracy of the measurement system.

In figure 7 , we show the results of an equipotential calculation [8] for the notched, parallel-plate electrode system. Symmetry is assumed about the center of the notch. The results of this plot show the field reduction at the full depth of the notch. Experimentally, the electro-optical observations of such a low-field region will not show a change in light intensity as the electric field increases in the gap.

For the measurements reported here, the interface material was $3.2 \mathrm{~mm}$ ( $1 / 8 \mathrm{in})$ thick pressboard which was soaked in $120^{\circ} \mathrm{C}$ transformer oil overnight. Two thicknesses were used to separate the plates by $6.4 \mathrm{~mm}$. The cell was heated to $106^{\circ} \mathrm{C}$ and dc voltage was applied. Upon the application of voltage, bubbles were observed to be emitted by the pressboard -- but only when voltage was applied. Because of these bubbles, the cell would not withstand $20 \mathrm{ky}$ initially. After a few days, the bubbling stopped and $30 \mathrm{kV}$ dc could be applied for short periods. Unfortunately, $30 \mathrm{kV}$ is really a minimum voltage for obtaining 


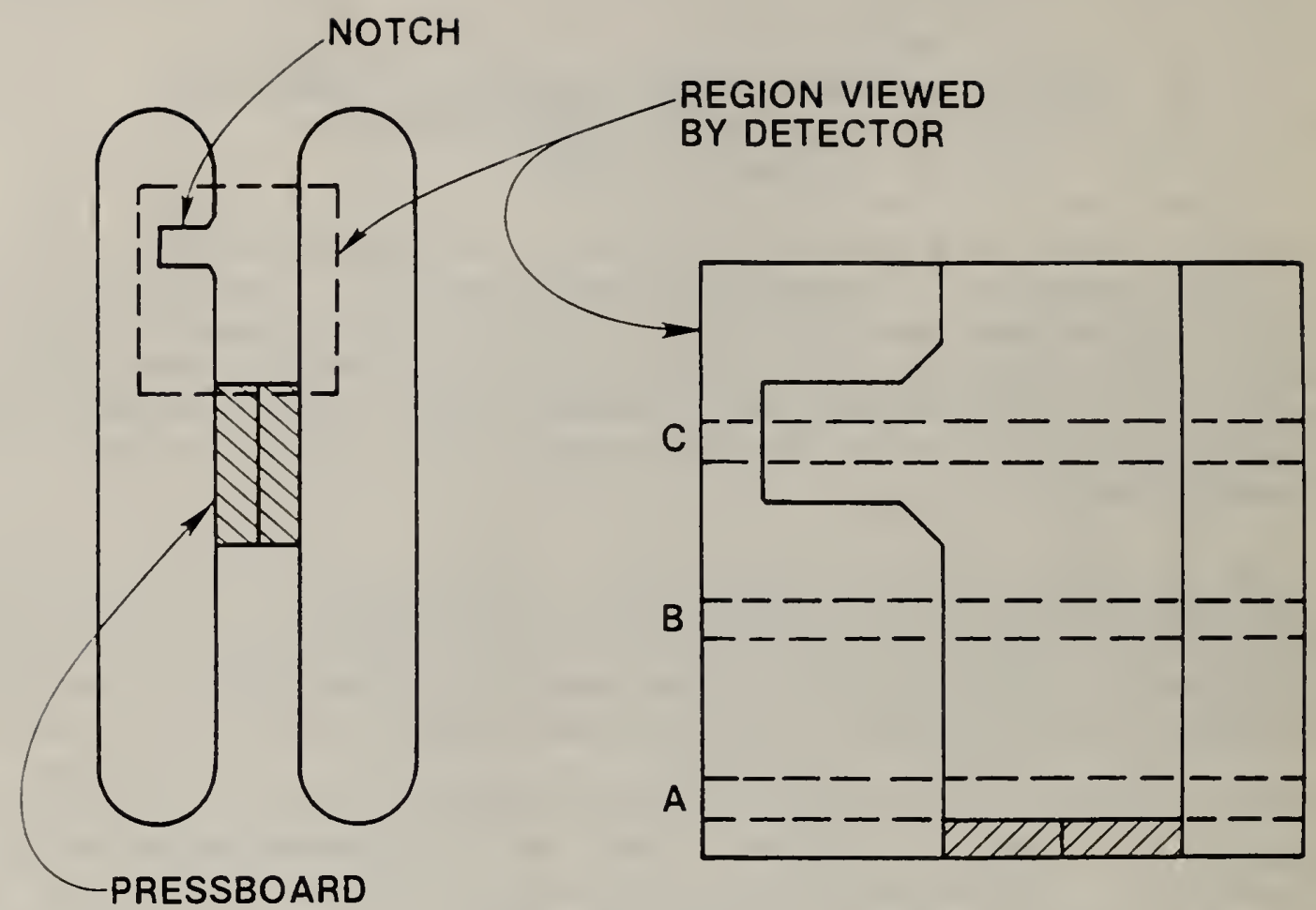

Figure 6. Electrode geometry with pressboard interface as viewed from the detector. This is an end view of the $\sim 30 \mathrm{~cm}$ plates with a pressboard interface extending $28 \mathrm{~cm}$ of the length. The notch in the electrode serves to produce almost zero field region. The light intensity as a function of position along different tracks $A, B$, and $C$ can be measured by the detector and compared with one another to obtain relative electro-optical field measurements. 


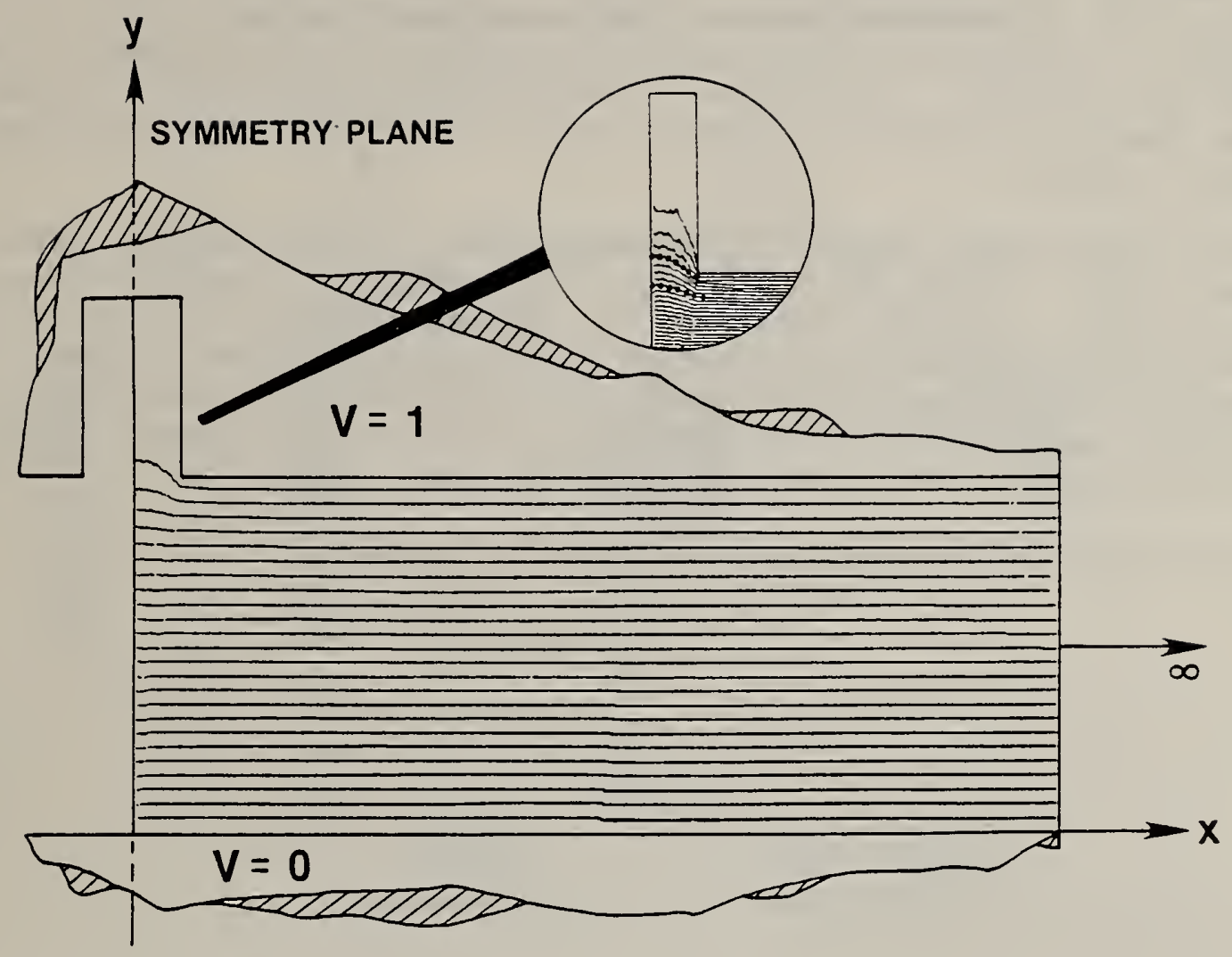

Figure 7. Computer calculation of the equipotentials for a notch in a parallel-plate electrode system assuming no space charge. The field is seen to be unaffected along the plate opposite the notch and essentially zero at the interior of the notch. The inset shows higher resolution of equipotentials where the dotted equipotentials are the ones shown in the main drawing. 
accurate field measurements with a $6 \mathrm{~mm}$ gap $(50 \mathrm{kV} / \mathrm{cm})$ because of the small Kerr effect in transformer oil. For larger fields, it will be necessary to develop better interface-preparation and liquid-handling methods.

To enhance the observability of the Kerr effect, the transformer oil was doped with nitrobenzene. Electro-optical observations were made along a strip directly above the interface and a strip about one gap spacing above the interface -- tracks $A$ and $B$ in figure 6 . The relative electric field as a function of position between the electrodes for these two strips is shown in figure 3 . To within 10 percent of the average field, the fields have the same spatial distribution. Based on our previous calculations [9], it is possible to show that a 10 percent field shift would represent a uniform surface charge of about $3 \mathrm{nC} / \mathrm{cm}^{2}$.

During the next quarter, these measurements will be refined in the following ways. First, the usefulness of the notched (zero field) area in improving the accuracy of the electric field measurements will be evaluated quantitatively. Second, the effect of various techniques to prepare test samples will be evaluated to determine the relationship between sample preparation and electric field distortion. Finally, the optical system will be improved to permit more accurate and more precise field measurements.

For further information, contact Dr. E. F. Kelley, (301) 921-3121. 


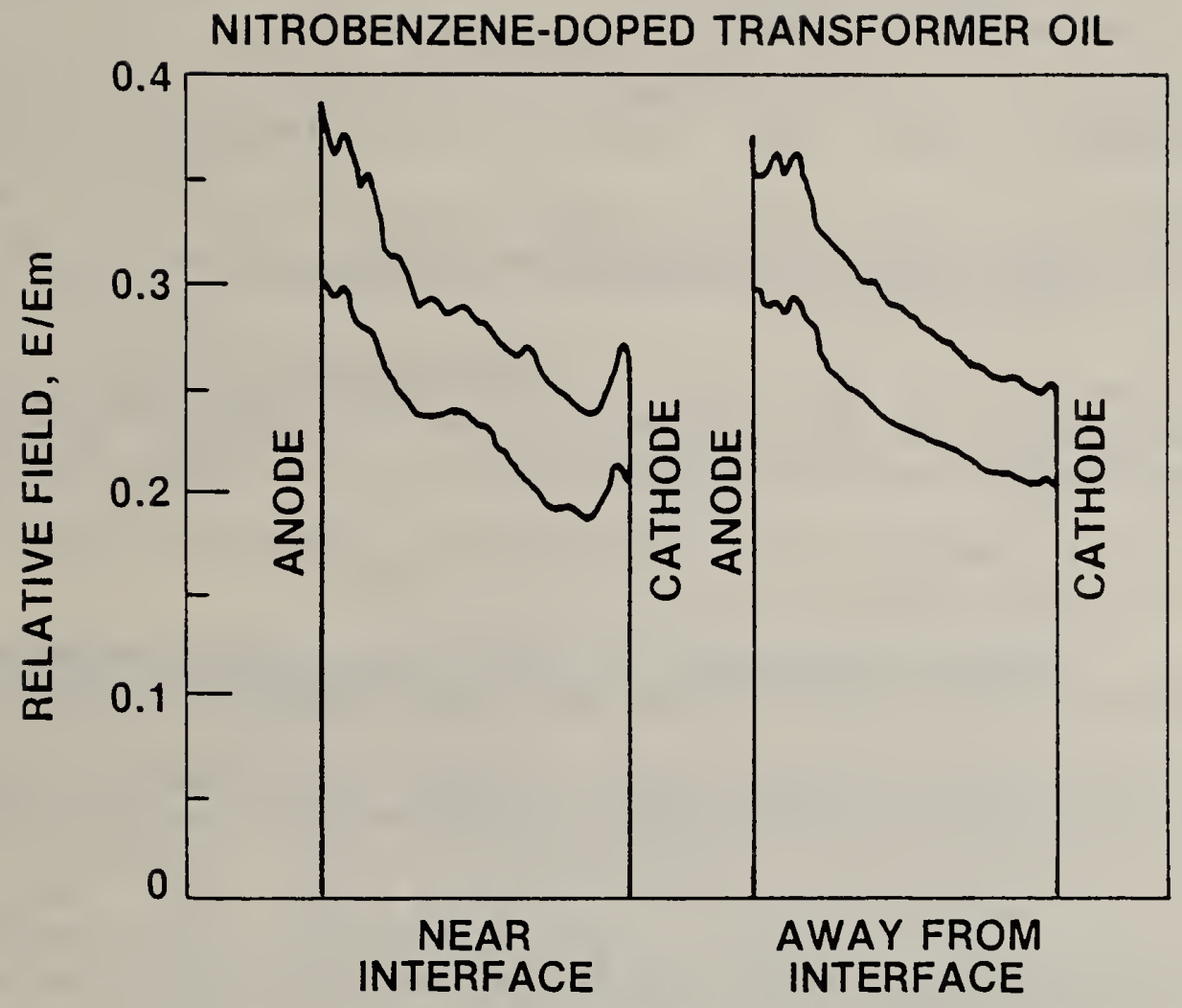

Figure 3. Comparison of electric field next to the interface and away from the interface in nitrobenzene dopped-transformer oil. Although there is an obvious, negative-space-charge-induced field distortion which is spread somewhat uniformly throughout the gap, the field distortion is not different near the interface from what it is away from the interface (with in 10\%). 


\section{REFERENCES}

[1] R. H. Mcknight, F. R. Kotter, M. Misakian, and J. Hagler, 1981 Annual Report: Electric and Magnetic Field Measurements, Nat. Bur. Stand. (U.S.), NBSIR 82-2527, 48 pages, July 1982.

[2] H. J. White, Industrial Electrostatic Precipitation, Addison Wesley, Reading, Mass. 1963, p. $126 \mathrm{ff}$.

[3] R. J. Van Brunt and D. Leep, Characterization of Point-Plane Corona Pulses in SF6, J. Appl. Phys., Vol. 52, No. 11, pp. 6588-6600, Nov. 1981.

[4] R. J. Van Brunt and M. Misakian, Mechanisms for Inception of DC and 60- Hz AC Corona in SF 6 , IEEE Trans. Elec. Insul., Vol. EI-17, No.2, pp. 106-120, Apr. 1982 .

[5] Y. A. Sahar, A. H. Qureshi, and N. H. Malik, Impulse Corona in Positive Rod-Plane Gaps Filled with SF 6 Gas Mixtures, Proc. 1982, [EEE Int'1 Symp. on Elec. Insul., pp. 197-201, June 1982.

[6] B. M. Smirnov, Negative Ions, translated by S. Chomet and edited by H. S. W. Massey, McGraw Hi11, pp. 148-168, 1982.

[7] P. J. Hay, The Relative Energies of $\mathrm{SF}_{6}^{-}$and $\mathrm{SF}_{6}$ as a Function of Geometry, J. Chem. Phys., Vol. 76, No. 1, pp. 502-504, Jan. 1982.

[8] Fortran subrouting PLTMG developed by R. Bank and A. Sherman of the University of Texas, and amplified and modified by J. Blue and C. Wilson of NBS.

[9] E. F. Kelley and R. E. Hebner, 1981 Annual Report: Optical Measurements for Interfacial Conduction and Breakdown in Insulating Systems, Nat. Bur. Stand. (U.S.), NBSIR (in press). 
1. PUBLICATION OR REPORT NO.

NBSIR $82-2586$
2. Performing Organ. Report Nof 3. Publication Date

October 1982

BIBLIOGRAPHIC DATA

SHEET (See in structions)

4. TITLE AND SUBTITLE

Development of Power System Measurements -- Quarterly Report

April 1, 1982 to June 30, 1982

5. $A U T H O R(S)$

R. E. Hebner, Editor

6. PERFORMING ORGANIZATION (If joint or other than NBS, see instructions)

NATIONAL BUREAU OF STANDARDS

DEPARTMENT OF COMMERCE

WASHINGTON, D.C. 20234

7. Contract Grant No.

T.0. No. 137

8. Type of Report \& Period Covered

$4 / 1 / 82-6 / 30 / 82$

9. SPONSORING ORGANIZATION NAME AND COMPLETE ADDRESS (Street, City. Stute, ZIP)

Department of Energy

Division of Electric Energy Systems

1000 Independence Avenue, S.W.

Washington, DC 20585

10. SUPPLEMENTARY NOTES

Document describes a computer program; SF-185. FIPS Software Summary, is attached.

11. ABSTRACT (A 200-word or less foctual summary of most significont information. If document includes a significant bibliogrophy or literature survey, mention it here)

This report documents the progress on four technical investigations sponsored by the Department of Energy and performed by the Electrosystems Division, the National

Bureau of Standards. The work described covers the period Aprit 1, 1982 to June 30, 1982. This report emphasizes the errors associated with measurements of space charge near dc transmission lines, the measurement of $r f$ attenuation in distribution cables, the characteristics of positive and negative corona in compressed $S F_{6}$ gas, and the measurement of the space charge density in transformer oil subjected to $60-\mathrm{Hz}$ excitation.

12. KEY WORDS (Six to twelve entries; alphabetical order; capitalize only proper nomes; ond separate key words by semicolons) cables; dc fields; high voltage; incipient fault; insulation; SF 6 ; space charge; transformer $0 i 1$.

13. AVAILABILITY

X] Unlimited

14. NO. OF

PRINTED PAGES

For Official Distribution. Do Not Release to NTIS

Order From Superintendent of Documents, U.S. Government Printing Office, Washington, D.C. 20402.

15. Price

¿ Order From National Technical Information Service (NTIS), Springfield, VA. 22161 

